\title{
Comparison between Face-to-Face Teams and Virtual Teams with Respect to Compliance with Directives
}

\author{
Daphna Shwartz-Asher ${ }^{1}$, Niv Ahituv ${ }^{2}$ \\ ${ }^{1}$ The Technion, Haifa, Israel \\ ${ }^{2}$ Tel Aviv University, Tel Aviv, Israel \\ Email: Sdaphna@technion.ac.il, ahituv@tauex.tau.ac.il
}

How to cite this paper: Shwartz-Asher, D. and Ahituv, N. (2019) Comparison between Face-to-Face Teams and Virtual Teams with Respect to Compliance with Directives. Journal of Service Science and Management, 12, 549-571.

https://doi.org/10.4236/jssm.2019.124038

Received: June 8, 2019

Accepted: June 27, 2019

Published: June 30, 2019

Copyright ( 2019 by author(s) and Scientific Research Publishing Inc. This work is licensed under the Creative Commons Attribution International License (CC BY 4.0).

http://creativecommons.org/licenses/by/4.0/

\begin{abstract}
This article portrays a study where the compliance was measured in virtual vs. face-to-face (FTF) teams ${ }^{1}$. To measure the influence of the virtuality level on the team members' reaction to instructions, an experiment was designed, in which a team task with a set of instructions was given to 150 subjects who participated in virtual or non-virtual (FTF) task solving meetings. The study's main finding indicates that while the virtual team members showed a high degree of compliance with the directive to distribute the labor among themselves and to appoint a chairperson, the non-virtual team members showed a low degree of compliance. It seems that the use of the "formality" variable may explain the compliance of the virtual team members as opposed to the lower level of compliance among members of the non-virtual teams. The knowledge that communication technology influences the compliance atmosphere is a point that deserves further research.
\end{abstract}

\section{Keywords}

HRM, Virtual Team, Compliance, Obedience, Experiment

\section{Research Background}

Over the past few decades, organizational tasks have increasingly become more complex and multidisciplinary. This phenomenon has led to a transition from individual work to teamwork [1]. Many organizations now use teams for various ${ }^{1} \mathrm{~A}$ preliminary version of this article has been presented at 2014 International Conference on E-commerce and Contemporary Economic Development. Please see Shwartz-Asher (2014). The article presented here is an extension of that presentation and contains a more comprehensive description and discussion of the research. 
activities that used to be conducted by individuals in the past [2]. The use of virtual teams has also become more prevalent, due to the growing number of international organizations, offshore outsourcing, global companies, and the fast development of communication tools (hardware and software), yet there is still a gap in empirical research that addresses the effectiveness of virtual teams [3] and a need to analyze the direct and indirect antecedents of virtual team effectiveness [4]. Considering the growing phenomenon of digital cooperation, the traditional definition of team member "compliance" should be examined in a virtual environment, studying virtual team members' behavior and reaction to instructions, or the absence of instructions [5]. The article poses the question whether a face-to-face (hereafter-FTF) team behaves differently from a virtual team with respect to compliance to guidelines and instructions. This research contributes to a better understanding of "compliance" as a form of social influence in virtual teams and hones the differences between FTF and virtual teams in terms of compliance. Its practical conclusions may facilitate the way organizations should form and manage virtual teams to improve the teams' work and self-management in today's virtual world.

The literature review begins by briefly discussing the concept of virtuality, as well as the need to study social influences and particularly team members' compliance within a virtual setting due to a limited body of knowledge studying this topic. The compliance term will then be defined and the corporate literature within the study of compliance will be discussed, starting from Milgram's obedience studies to recent studies linking the formal element of corporate culture with employee obedience/compliance.

\subsection{Literature Review}

Technological advances, a globally distributed workforce, and a rapidly changing business context have created both the ability and need for organizations to operate over great distances [4]. Virtual teams are defined as interdependent individuals physically separated from one another and relying on information technologies to communicate, collaborate, and coordinate work to achieve common goals [6] [7] [8] [9] [10].

Managing virtual teams is different and more complex than managing FTF teams, yet virtual teams are still groups of individuals that share most of the characteristics and dynamics found with traditional teams. The effective management of virtual teams requires knowledge and understanding of the fundamental principles of team dynamics regardless of the time, space, and communication differences between virtual and FTF work environments [11]. Maynard et al. [12] propose that even for new team configurations, transactive memory systems and preparation activities are critical for effectiveness. Their findings suggest that preparation activities relate significantly to effectiveness as mediated by transactive memory systems. Furthermore, interdependence interacted with members' percentage of time allocated to the team as related to preparation ac- 
tivities. Hoch and Kozlowski [13] evaluated the impact of traditional hierarchical leadership, structural supports, and shared team leadership on team performance. Their findings indicated that the extent to which teams were more virtual attenuated relations between hierarchical leadership and team performance but strengthened relations for structural supports and team performance. However, shared team leadership was significantly related to team performance regardless of the degree of virtuality.

In a virtual team, members can have very different cultural backgrounds; they often physically work in different locations and are experts in distinct fields [14]. A team's virtuality level has become an integral part of the definition of a team [15], as many variables are affected by it. The lack of media richness, or opportunity for using non-verbal cues, leads to misunderstood communications and may limit the development of trust [16]. It has been shown that FTF team members are more cohesive [17], have stronger social ties [18], are more dedicated to the task and to other team members [19], have a stronger team identity [20], and have more affection towards the other team members [21] relative to virtual team members. While strong social ties can be achieved in virtual teams as well, it is known to take longer than with FTF teams [22].

Many researchers have attempted to find the reasons why virtuality has a negative influence upon team output. Some of the reasons discussed in research literature include frequency of interaction and physical distance [23], the fact that team members are not familiar with one another on a personal level [24], difficulty in sharing information, and insufficient and confusing discussions [25]. Maruping and Agarwal [26] show that teams tend to use different sorts of communication technologies for different kinds of interpersonal interaction. Another group of researchers compared the impact of various communication technologies on corporate teams, assuming that some technologies limit information transference [27]. The comparisons concluded that FTF teams are more efficient than teams that use video communication [28], and that video communication is more efficient than audio communication [29]. Moreover, the addition of text alongside video or audio communication improves performance [30], and satisfaction [31].

Caya et al. [4], who reviewed and synthesized one hundred and twenty-one empirical studies of virtual teams published since 1990, developed a framework for understanding virtual team dynamics. They analyzed the direct and indirect antecedents of virtual team effectiveness and identified key gaps in both their knowledge of, and approach to studying, virtual teams. They pointed at interpersonal states as affecting teams and at the understudied area involves the links between interpersonal, task, and IT-related issues.

Since virtual team members don't share time, space, culture, and identity, their degree of compliance could be influenced by many variables, such as weak cohesiveness and social ties, or high autonomy and interdependence. By studying compliance of team members in virtual setting, our hope is to fill in a part of this understudied area of social influences. 
Compliance, obedience, and conformity are the three forms of processes which can affect the way an individual behaves in a social setting, all the way from following fashions and unwritten social norms, to committing immoral acts just because the individual was ordered to do so by someone within a position of authority. Compliance refers to a form of social influence in which an individual give into expressed requests from another person or other people. Obedience refers to a form of social influence in which a person gives into express instructions or orders from an authority figure without question, or as acting in accordance with rules or orders [32]. Conformity refers to a trait that makes people change their behavior to fit social norms and behave according to the wishes of others [33]. There are many common aspects between compliance, obedience and conformity; however, there seem to be more similarities between compliance and obedience than those shared by conformity.

Song et al. [34] defined compliance as responding to a request made by others. Conformity, which is a major determinant of compliance, occurs when the subject demonstrates the same behavior or attitude as the object. In corporate teams, the subject is the individual who conforms. The object(s) may be individuals, groups, organizations, policies, rules, and regulations, or the experience or natural instinct of the subject. Conformity is traditionally divided into two categories: irrational conformity (herd behavior) and rational conformity (abidance, compliance, and obedience).

Cadsby et al. [35] examined authority in relation to compliance and demonstrated that simply telling people that they are required to pay a tax produces remarkably high compliance rates and less sensitivity to changes in economic variables than in an earlier experiment, which used language pertaining to an invitation-to-gamble. This suggests that many people pay taxes despite the financial attraction of non-compliance because they are strongly inclined towards obeying authority.

Russell and Gregory [36] examined a variety of work influences according to Milgram's [37] obedience studies in a controlled laboratory, to aid the understanding of how functionaries may resolve moral dilemmas in a bureaucratically organized work situation. By doing so, they provided an alternative explanation for Milgram's new baseline findings, one that is more consistent with Bandura's concept of "moral disengagement" [38], and Barnard's concept of an organizational "zone of indifference." They concluded that Milgram's experiments have less to do with compliance and obedience to authority per se, and more to do with how people resolve moral dilemmas, those they are confronted by in a structured organizational setting.

The organizational setting is, in many ways, equal to organizational culture. The organizational culture's perspective, therefore, should be included in the discussion of compliance. Interligi [39] proposed a framework that captures the complexities of the relationship between compliance and culture, by drawing from two theories relevant to organizational culture: The Neo-Institutional Theory and the Competing Values Model. By integrating elements of these theo- 
ries, three core dimensions of compliance culture are identified: legitimacy, permeability, and control. These core dimensions provide for a pluralist approach to theorizing about the compliance culture. One basic component of organizational culture is "top management". Hu et al. [40] showed how top management can play a proactive role in shaping employee compliance behavior in addition to the deterrence-oriented remedies advocated for in existing literature and have refined theories pertaining to the role of organizational culture in shaping employee compliance behavior.

Cultural compliance is another main component in the exploration of compliance. One of the studies of cultural compliance is that of Alanazi and Rodrigues [41] who examined cultural compliance and found that cognitive and affective reactions of Saudi students that were examined with respect to compliant behaviors (leading to a good outcome or a bad outcome) were affected by six power bases. As they hypothesized, power bases had significant effects on compliant behaviors. However, when the outcome of the compliant behavior was bad, the compliant behavior caused by a coercive influence led to the perception of more internality and controllability. They also found that the perception of less internality and controllability of compliant behaviors was caused by an informational influence.

Formality (versus informality) characteristics of organizational culture should also be examined since they influence compliance. Wright [42] shows that there is a potential relationship between formal training delivered to staff and the self-perceived level of records management competency, namely the more training the staff receive, the more the staff perceives the need for further training, and the greater the level of compliance with records management.

In conclusion, previous studies have dealt with compliance of team members as well as with virtual teams. However, the impact of virtuality on team members' compliance has not been researched. Knowing the relationship between the two factors is very important in an era where a large amount of team functions is carried out through long distance communications, either audio or visual. It will be shown later that some of the results are counter intuitive, which makes it more important to managers of virtual team operations.

\subsection{Research Hypotheses}

Structuredness, in the current study, means to be instructed, in writing, how to work according to a recommended working procedure dictated in advanced. It includes, among others, a request to appoint one of the members as a spokesperson, another one as an information coordinator, and, finally a chairperson who will be in charge of assembling the information and reporting. This structure reflects a desire to work according to certain guidelines: to work according to a recommended procedure, to divide the labor and to appoint a leader. It is assumed that the organizational structure components adopted by the subjects reflect compliance and that, since the directives are given for the benefit and the 
success of the team, there is no reason for the subjects to ignore it.

A major issue that was investigated in this study was whether there is a difference between the FTF and the virtual teams in that respect. Altogether, we title this as compliance.

The following hypotheses concern several of the issues dealt with by the team members.

\subsubsection{Work Processes}

In large teams, meeting procedures are usually better defined and often include better technological coordination than in small groups [43]. Studies show that successful teams focus on the task [44], on structured goals [17], and on the development of routine [45]. Successful teams also take the time to understand the process and contents of the work [46], especially in its initial stages [47]. However, once guidelines are not given, it can be assumed that the team members will not necessarily initiate certain measures (process) and therefore:

H 1.1 Structured team members will work according to the recommended process, as requested (i.e., will show a high degree of compliance), while unstructured team members will not work according to a recommended process (i.e., will show a low degree of compliance).

In virtual teams, not only does the medium limit the team's ability to coordinate information [48], but a great deal of time is spent on understanding how to execute the task [49]. Additionally, in virtual team meetings, the members are physically distant from each other and, therefore, take longer to coordinate than during FTF team meetings [50]. Coordination processes are positively related to performance and satisfaction [51] and become more significant to the team outcomes as time passes [52].

According to Milgram's [37] study participants are more compliant when the person providing the instruction is physically present, in comparison to a situation in which the instruction is given by phone (or other similar electronic communication medium). Accordingly, it is conceivable that virtual communication, which potentially distances the person giving and receiving instruction, in comparison to FTF communication, will reduce compliance levels. Respectively, compliance rates will be higher among FTF team members, therefore:

$\mathrm{H}$ 1.2 Members of structured virtual teams will not work according to a recommended process (i.e., will show a low degree of compliance), while structured non-virtual team members will work according to the recommended process (i.e., will show a high degree of compliance).

\subsubsection{Division of Labor}

The division of labor does not have a direct influence on team performance, yet it does have an indirect influence, by means of perceived efficiency and team coordination [53] and is a stronger predicating variable than individual characteristics [54]. The use of expertise aids in reducing errors [55], and functional diversity and is, therefore, important for achieving team efficiency [56]. A team 
that is structurally diverse is a team whose members hold different positions or tasks, are distributed in various branches, and can, therefore, be exposed to unique information. In this manner, sharing unique external information elevates performance [57]. Therefore:

H 2.1 Structured team members will divide the labor between them, as requested (i.e., will show a high degree of compliance), while unstructured team members will not divide the labor between them (i.e., will show a low degree of compliance).

Regarding to Milgram's [37] argument, virtual communication, which potentially distances the person giving from the ones receiving instruction, in comparison to FTF communication, will reduce compliance levels. Respectively, compliance rates will be higher among FTF team members, therefore:

H 2.2 Members of structured virtual teams will not divide the labor between themselves (i.e., will show a low degree of compliance), while structured non-virtual team members will divide the labor between themselves, as requested (i.e., will show a high degree of compliance).

\subsubsection{Hierarchy}

To explain the importance of the manager in integrative groups, Maier [58] compared the group to a starfish and the group's manager to the central nervous system of the starfish. When individuals act as an organized unit, they become a higher type of organization-a single whole organism. Even when there is no formal division of labor, the role of the manager is divided between the team members [59]. When there is a formal manager, status labels have a strong effect on the team members as being an authoritative figure [60]. Therefore:

H 3.1 Structured team members will appoint a chairperson, as requested (i.e., will show a high degree of compliance), while unstructured team members will not appoint a chairperson (i.e., will show a low degree of compliance).

A series of studies has shown that effective virtual management/leadership is dependent on the communication ability within the team [61] [62] [63]. It has been shown that in virtual teams, managers/leaders send more (and longer) messages than in FTF teams [64]. Our assumption, consistent with Milgram's [37] studies, is that the virtual setting will reduce compliance. Therefore:

H 3.2 Members of structured virtual teams will not appoint a chairperson (i.e. will show a low degree of compliance), while structured non-virtual team members will appoint a chairperson, as requested (i.e. will show a high degree of compliance).

\subsubsection{Hypotheses Summary}

H $1(1.1,2.1,3.1)$ concerning structured team members: Structured team members will work according to the recommended process, will divide the labor between them, and will appoint a chairperson, as requested (i.e., will show a high degree of compliance), while unstructured team members will not work according to a recommended process, will not divide the labor between themselves, and 
will not appoint a chairperson (i.e., will show a low degree of compliance).

H $2(1.2,2.2,3.2)$ concerning virtual teams members: Members of structured virtual teams will not work according to a recommended process, will not divide the labor between themselves, and will not appoint a chairperson (i.e., will show a low degree of compliance), while structured non-virtual team members will work according to the recommended process, will divide the labor between themselves, and will appoint a chairperson, as requested (i.e., will show a high degree of compliance).

The literature review above, embedded with our main hypotheses, has discussed the concepts of virtuality, compliance, and team structural properties. The following section describes the methodology we have used to examine compliance among virtual teams.

\section{Methodology}

An experiment was designed in which a team task was given to 150 undergraduate students in an academic institute. The study subjects were grouped into teams of three. They had to share information in order to complete a task that took approximately 30 minutes to complete. The research design was a $2 \mathrm{X} 2$ between subjects' factorial design: the two factors were team structure (structured vs. unstructured) and mode of communication (virtual vs. FTF). The research design included four experimental conditions (see Table 1). Each condition was implemented on 12 - 13 teams (four conditions X 12 - 13 teams $=50$ teams. 50 teams X 3 subjects $=150$ subjects).

The subjects were students within the same academic unit, however, they belonged to different student cohorts, therefore were not familiar with each other and it was unlikely that they had any previous virtual or FTF interaction among them.

Though today's students are tomorrow's employees, often a concern is raised regarding the link between student teams and professional teams. In their analysis of more than 200 articles relating to experimental research involving group support systems, Fjermestad and Hiltz [65] report that 94 percent of those studies involved students as subjects. Souren, Samarah, Seetharaman, and Mykytyn [66] claimed that, since previous research found no significant differences between managers and students, they felt comfortable with the background of their student subjects. Shuman, Besterfield-Sacre and McGourty [67] also pointed at the link between undergraduate student teams and professional workforce teams,

Table 1. Experimental conditions.

\begin{tabular}{cccc}
\hline $\mathrm{N}$ & Virtuality level & Structural level & Structure and mode of communication \\
\hline 12 & 0 & 0 & FTF-unstructured team \\
12 & 0 & 1 & FTF-structured team \\
13 & 1 & 0 & Virtual-unstructured team \\
13 & 1 & 1 & Virtual-structured team
\end{tabular}


claiming there are a number of creative ways that skills (communication, teamwork, ethics...) can be learned, particularly at engineering institutions that aim to teach and reinforce outcome combinations. In this perspective, studying social influence and particularly compliance can be studied as an important teamwork skill that will benefit virtual team members.

\subsection{Procedure}

Subjects in groups of three were invited to meetings (virtual or non-virtual) that were conducted using MSN-Messenger or FTF communication. The virtual team met only virtually, not FTF at all. The virtual and non-virtual appointments were scheduled by the experimenter who attended and recorded the meetings. She physically attended the non-virtual meetings held in the college facilities (classes), and virtually attended the virtual meetings using her computer device, as did the virtual team members.

The process for all conditions of the experiment included an intellectual task. Each team member received a discrete piece of verbal information, where only the aggregation of all of the information together revealed the whole "picture" and led to the correct solution (based on Stewart \& Stasser [68] murder mystery).

As a motivational factor, a prize was offered (in advance) to the members of the team that solved the mystery the fastest among the teams that handed a correct solution.

The structured condition of the experiment included a preliminary manipulation, namely, instructions in writing to the team members asking them to nominate a chairperson who would be in charge of assembling the information, and to appoint a spokesperson and an information coordinator for the team. In addition, a recommended work procedure was given to the team members. The recommended procedure included instructions to find anchor information at the first stage of the problem-solving process, to locate the related information at the second stage, and to complete the missing information gap at the third and final stage. This preliminary manipulation did not occur in the non-structured condition.

\subsection{Operationalization of the Dependent Variables}

All compliance dependent variables were dichotomized variables, which measured the level of individual participant compliance regarding a given structural directive:

1st compliance component: worked by process or did not work by process.

2nd compliance component: divided the labor or did not divide the labor.

3rd compliance component: appointed a chairperson or did not appoint a chairperson.

The statements "Worked by process", "Divided the labor" and "Appointed a chairperson" were collected, following the experiment, via a short survey the 
team members filled in (see Appendix A) as statements referring to their self-perception of the task.

The next section presents the findings regarding the hypotheses.

\section{Findings}

The compliance data collected in this study was categorical and dichotomous.

We tested our hypotheses concerning differences in proportion by Fisher's exact tests.

Chi-squared test requirements were not met, therefore no test for the homogeny of the odds ratio was implemented.

\subsection{Work Processes (Table 2)}

An insignificant difference was found between the structured and the non-structured team members' outcomes: Structured team members, as well as unstructured team members, worked according to a recommended process.

Therefore, H 1.1 is not supported.

Yet, among virtual team members, a close to significant difference $(\mathrm{p}=0.058)$ was found between the (26) non-structured virtual team members who worked according to process, in comparison to the (34) structured virtual team members who worked according to process.

An insignificant difference was found between the structured non-virtual team members and the structured virtual team members who worked according to process: Members of structured virtual teams, as well as structured non-virtual team members, worked according to the recommended process.

Therefore, $\mathrm{H} 1.2$ is not supported.

However, among the non-structured team members, a significant difference $(\mathrm{p}=0.003)$ was found between the (26) non-structured virtual team members who worked according to process, in comparison to the (34) non-structured non-virtual team members who worked according to process.

\subsection{Division of Labor (Table 3)}

A significant difference $(\mathrm{p}<0.0005)$ was found between the structured and the non-structured team members' outcomes: Structured team members divided the

Table 2. "Working according to process" Compliance level by experimental conditions.

\begin{tabular}{|c|c|c|c|c|}
\hline & \multicolumn{4}{|c|}{ Structural Component } \\
\hline & \multicolumn{2}{|c|}{0 Not given } & \multicolumn{2}{|c|}{1 Given } \\
\hline & \multicolumn{4}{|c|}{ Virtuality } \\
\hline Compliance & 0 Non Virtual & 1 Virtual & 0 Non Virtual & 1 Virtual \\
\hline 1 worked according to process & 34 & 26 & 31 & 34 \\
\hline 0 didn't work according to process & 2 & 13 & 5 & 5 \\
\hline $\mathrm{N}$ & 36 & 39 & 36 & 39 \\
\hline
\end{tabular}


Table 3. "Division of labor" Compliance level by experimental conditions.

\begin{tabular}{cccccc}
\hline \multicolumn{5}{c}{ Structural Component } \\
\hline \multicolumn{5}{c}{ 0 Not given } & \multicolumn{3}{c}{ V Given } \\
\hline Compliance & 0 Non Virtual & 1 Virtual & 0 Non Virtual & 1 Virtual \\
\hline 1 divided the labor & 3 & 3 & 22 & 35 \\
0 didn't divide the labor & 33 & 36 & 14 & 4 \\
$\mathrm{~N}$ & 36 & 39 & 36 & 39 \\
\hline
\end{tabular}

labor between them (i.e., showed a high degree of compliance), while unstructured team members didn't divide the labor between them.

A significant difference $(\mathrm{p}<0.0005)$ was also found between the non-virtual non-structured team members and the non-virtual structured team members, and a significant difference $(\mathrm{p}<0.0005)$ was found between the virtual non-structured team members and virtual structured team members.

Therefore, H 2.1 is supported.

A significant difference $(\mathrm{p}=0.006)$ was found between the structured virtual and the structured non-virtual team members' outcomes: (35) members of structured virtual teams divided the labor between themselves (i.e., showed a high degree of compliance), while (22) members of structured non-virtual teams divided the labor between themselves.

Therefore, $\mathrm{H} 2.2$ is not supported.

\subsection{Hierarchy (Table 4)}

A significant difference $(\mathrm{p}<0.0005)$ was found between the structured and the non-structured team members' outcomes: Structured team members appointed a chairperson (i.e., showed a high degree of compliance), while unstructured team members didn't appoint a chairperson.

A significant difference $(\mathrm{p}<0.0005)$ was also found between the non-virtual non-structured team members and the non-virtual structured team members, and a significant difference $(\mathrm{p}<0.0005)$ was found between the virtual non-structured team members and virtual structured team members.

Therefore, H 3.1 is supported.

A significant difference $(p=0.002)$ was found between the structured virtual and the structured non-virtual team members' outcomes: (36) members of structured virtual teams appointed a chairperson (i.e., showed a high degree of compliance), while (22) members of structured non-virtual teams appointed a chairperson.

Therefore, H 3.2 is not supported.

\section{Discussion}

This article poses the question whether a face-to-face (FTF) team behaves differently than a virtual team with respect to compliance to guidelines and instructions. 
Table 4. "Hierarchy" Compliance level by experimental conditions.

\begin{tabular}{ccccc}
\hline & \multicolumn{5}{c}{ Structural Component } \\
\hline \multicolumn{5}{c}{ 0 Not given } \\
\multicolumn{5}{c}{ Virtuality } \\
\hline Compliance & 0 Non Virtual & 1 Virtual & 0 Non Virtual & 1 Virtual \\
\hline 1 appointed a chairperson & 1 & 2 & 22 & 36 \\
0 didn't appoint a chairperson & 35 & 37 & 14 & 3 \\
$\mathrm{~N}$ & 36 & 39 & 36 & 39 \\
\hline
\end{tabular}

The question is studied through an experiment focused on work processes, division of labor, and hierarchy.

\subsection{Work Processes}

Structured team members, as well as unstructured team members, worked according to a recommended process. It seems that working according to a required process, as part of the current task, was an obvious strategy for the non-virtual team members; therefore, the conduct of the team members who were not asked to work according to such a process should not be seen as being non-compliant but simply as the use of basic intuitive tools. In other words, the instruction to work according to a required process is not required, because the work is done through a process in any case, whether it is required or not.

Members of structured virtual teams, as well as structured non-virtual team members, worked according to the recommended process. This finding means that there is no compliance issue for the virtual or non-virtual team members, as hypostasized. Again, it seems that working according to a required process, as part of the current task, was a strategy that is reasonable to adopt if not suggested, as well as not reasonable to reject when suggested.

Yet, among non-structured team members, 26 (out of 39) of the virtual team members adopted the process without being asked to, in comparison to 34 (out of 36) of the virtual team members who adopt the process without being asked to, implying non-structured virtual team members did not concur with this basic intuitive tool as the non-structured non-virtual team members. Since this finding highlights the difference between teams who were not given directives, we suggest explaining that by a lack of intuitive tool usage among virtual teams, or a lack of feeling they need to adopt a systematic process, rather than compliance issues.

\subsection{Division of Labor}

Structured team members divided the labor between them (i.e., showed a high degree of compliance), while unstructured team members didn't divide the labor between them. Same pattern was found between the non-virtual non-structured team members and the non-virtual structured team members, as well as between 
the virtual non-structured team members and virtual structured team members.

This study's main finding indicates that while 35 (out of 39) members of structured virtual teams divided the labor between themselves (i.e., showed a high degree of compliance), only 22 (out of 36) members of structured non-virtual teams divided the labor between themselves (i.e., showed a high degree of compliance).

This finding stands in contrast to our (H2) hypothesis-members of structured virtual teams will not divide the labor between themselves (i.e., will show a low degree of compliance), while structured non-virtual team members will divide the labor between themselves, as requested (i.e., will show a high degree of compliance).

This finding is somewhat counter intuitive. One might initially assume that non-virtual teams will maintain more "self-discipline" since they sit together and are easier to control. However, the results indicate that the virtual teams are more disciplined. The question arising is: Are the virtual team members "too compliant"? Or, are the non-virtual team members "too non-compliant"? Either way, there is a gap in the level of compliance which must be explained. It can be speculated that the non-virtual team members showed a low degree of compliance due to some sort of self-confidence since they see each other and can follow not only the words but the body language and their own intuition. On the other hand, the virtual team members might feel some weakness to the distance among them, so they completed the directive verbatim. In other words, it is easier to exercise authority and cause compliance through use of computer-mediated communication.

One clue to understand the virtual team members' high degree of compliance vs the non-virtual team members' low degree of compliance might be that it easier to constitute a compliance culture by legitimacy, permeability, and control [39] in a virtual setting. Other clues can be found in the virtual team literature: physical distance [23], the fact that team members are not familiar with one another on a personal level [24], difficulty in sharing information, and insufficient and confusing discussions [25]. These are all virtuality negative influences on team output yet can explain a high degree of compliance.

Our view is that the differences in the compliance level as reflected in the disparity in the Division of Labor is caused by several accumulated reasons as seen in the aforementioned references. FTF team members have more confidence in their ability to achieve the goals since they can converse more easily, they watch how assertive is each of them, and they not only follow the spoken words but also watch the body language of the other members. Hence, they do not need the discipline that is exercised when the members are not sitting together. They are confident that they can accomplish the mission without a well-structured process. The virtual team members miss an important component of team communication: the nonverbal communication [51]. They believe that they must exercise a much more disciplined and structured way of communication; otherwise, the whole process might become chaotic. Consequently, they become more com- 
pliant.

Since use of computer-mediated communication interaction, the giver and the receiver of the directive are physically apart, compared to FTF communication in which the giver and the receiver of the directive are physically close to each other, the above finding may seem to contradict the findings of Milgram's classic study of obedience [37]. Milgram found that subjects are more compliant when the person giving the directive is physically present in the location, as opposed to a situation in which the directive is given by phone. This effect has been termed "white coat syndrome" by Mancia et al. [69] which showed that a subject's blood pressure rises temporarily in the presence of a doctor or nurse, but it is fine during home measurements.

An analysis of the current study findings makes sense considering the "formal" variable. If virtual communication (or computer-mediated communication) is a form of formal communication, while face to face communication is considered to be a form of informal communication, then the fact that the structured non-virtual team members who showed a low degree of compliance while the structured virtual team members who showed a high degree of compliance, can be understood. This explanation is also consistent with Mancia's "white coat syndrome" findings indicating that people tend to comply with formal authority more than with informal authority. In many ways, communication technologies, such as social networks, are the "white coats" of the present era.

\subsection{Hierarchy}

Structured team members appoint a chairperson (i.e., showed a high degree of compliance), while unstructured team members didn't appoint a chairperson. Same pattern was found between the non-virtual non-structured team members and the non-virtual structured team members, as well as between the virtual non-structured team members and virtual structured team members.

This study's main finding indicates that while 36 (out of 39) members of structured virtual teams appointed a chairperson (i.e., showed a high degree of compliance), only 22 (out of 36) members of structured non-virtual teams appointed a chairperson (i.e., showed a high degree of compliance). In other words, many of the non-virtual team members who were asked to appoint a chairperson did not do so, as opposed to the virtual team members.

This finding stands in contrast to our (H2) hypothesis-Members of structured virtual teams will not appoint a chairperson (i.e., will show a low degree of compliance), while structured non-virtual team members will appoint a chairperson, as requested (i.e., will show a high degree of compliance).

This is the time to repeat the question raised in the previous section (regarding division of labor): Are the virtual team members "too compliant"? Or, are the non-virtual team members "too noncompliant"? Here too, we argue that the explanation for the gap in the level of compliance is similar to the one discussed 
in the previous section: a mechanism which gives a feeling of power to the non-virtual team members, or a feeling of weakness to the virtual team members, since they completed the directive verbatim. In other words, the examination of the team members completing the task of appointing a chairperson also indicates that it is easier to exercise authority and cause compliance through use of computer-mediated communication, while use of FTF communication causes less compliance.

The effective management of virtual teams requires knowledge and understanding of the fundamental principles of team dynamics regardless of the time and space [11]. However, compliance culture might develop to compensate for time and space limitation, in order to create shared behavior. Since top management can play a proactive role in shaping employee compliance behavior [40], an explanation to the virtual and non-virtual compliance levels can be found in generating management roles in the different settings-the traditional vs. the virtual one.

Similar to the division of labor issue, when appointing a chairperson, the use of the "formality" variable may also explain the compliance of the structured virtual team members as opposed to the low degree of compliance among members of the structured non-virtual team members. These findings are consistent with findings from previous studies according to which people tend to comply with formal authority more than with informal authority.

\section{Conclusions}

We will discuss first the theoretical conclusions and then mention some practical conclusions. The current study attempts to examine compliance among virtual team members, an issue that, to the best of our knowledge, has not yet been discussed in the pertinent literature. For this purpose, an experiment was designed, in which an intellectual team task was given to 150 subjects, who were grouped into teams of three and had to share information in order to complete the task. The groups were divided into virtual and non-virtual ones, and into structured and non-structured ones. The structured groups of the experiment received instructions in writing, asking them to follow a recommended work procedure: to appoint a spokesperson and an information coordinator and to nominate a chairperson who would be in charge of assembling the information. This preliminary manipulation was not provided to the non-structured groups.

The main finding of this study indicates that while the virtual team members showed a high degree of compliance with the directives (i.e., to divide the labor between them and to appoint a chairperson), the non-virtual team members showed a low degree of compliance. This finding is counter-intuitive. One would expect that when members "see" each other, they would be more obedient and compliant. It seems, though, that there is a mechanism, which provides a feeling of power to non-virtual team members in comparison to the virtual team members. This mechanism can be the power of formality: virtual communication is 
perceived as more formal than FTF communication, and in today's information era it functions as the "white coats" of Mancia's studies, i.e., increasing the authority of directives given and the compliance with these directives.

For business or public organizations that form and operate virtual teams (and there are many thousands of those), the looming practical conclusion is that there are disparities between the behavior of members of a virtual team and members of an FTF team. A major difference is in the compliance behavior. Thus, if management wishes to appoint a certain member to lead a team or to perform a certain function in the team, it should do so in advance. Otherwise, in a virtual team someone else might take that role, and in an FTF team, the team will mostly base its work on informal relationships and no one will formally take the required function.

Regarding study limitations, first, we would like to refer to the research method. Experiments, by definition, are conducted in a controlled environment. Furthermore, the subjects in our experiments were undergraduate students, a group that does not necessarily reflect the participants in organizational task teams, and often a concern is raised regarding the use of students as subjects [66]. Additionally, as there is a wide spectrum of research methods that can be used to examine the proposed research model such as surveys and case studies, we believe that examination of the proposed research model with additional research methods will assist in reinforcing it. Using such methods would assist in examining the conceptual model in different settings and countries.

Second, the experiments were performed by using a specific task (an intellectual task) with a specific form of communication (MSN messenger) and specific team (ad-hoc). Straus and McGrath [27] argue that forms of communications that can transfer more social cues increase performance and satisfaction. Since we didn't consider long-term teamwork, or any type of teams that need time to develop, it is recommended to examine the research model by using additional forms of communications, tasks, and teams.

Third, in the presented experiment, the compliance level was measured by the team members agreement among two or more (out of three): if two or three members stated that they worked by process, divided the labor, and appointed a chairperson, we counted it as a high level of compliance. Yet the assumed dichotomized "yes or no" relationship doesn't exist in reality. Working in the virtual sphere may moderate the process and have some impact on the outcomes, but it won't be the cause for "being compliant" or not. It would benefit if the compliance attitude were to consider using Likert Scales which have more nuance, rather than simple dichotomized "yes or no" measures.

Finally, the level of analysis should be examined. Although this study measured compliance among individuals, the individuals were required to collaborate as a team in a joint exercise. It would be interesting to examine compliance among individuals who are not required to collaborate with others, similarly to Milgram's studies, and alternatively, to examine compliance among groups that 
are regularly required to collaborate.

Compliance among virtual teams is a topic, which, to the best of our knowledge, has not previously been mentioned in research literature. Therefore, the first contribution of this study is to observe these contracts within one experimental setting and to discuss their mutual interaction.

Our findings are somewhat counter-intuitive. They indicate that under certain conditions, virtual communication increases compliance in comparison to FTF communication. This conclusion should be recognized by those wishing to manage others. The knowledge that communication technology influences the compliance atmosphere is a point that deserves further research, under different communication technologies and working environments.

One innovation of this study is the connection between the "white coats" effect of Mancia's studies and between computer-mediated communications phenomena, which appear, according to our findings, to increase the subjects' compliance in relation to FTF communication.

It might be the written word that characterized the virtual environment (in contrast to the spoken one) that contributes to the compliance atmosphere we observed, referring to two (Division of labor and Hierarchy) out of our three research variables. We explain the automatic use of the third variable (Work process) as influenced by the common knowledge that it is simplifying the teamwork to manage the process. Yet we hypothesize that other structural instructions will act the same as Division of labor and Hierarchy-meaning they will be more obeyed in a virtual environment than in an FTF one. These hypotheses can be tested in future research in which structural team variables such as regulation, decision-making procedures, team progress monitoring, conflict resolution, timing, opening and closing procedures, ground rules of operation will take place.

One aspect of virtual teams that had not been addressed in traditional definitions was the degree of technology-mediation, as opposed to FTF interaction, that is necessary for a team to be considered virtual. For example, some researchers specifically state that virtual teams are teams that interact exclusively through electronic media and, therefore, exclude teams that meet FTF. On the other hand, several researchers have relaxed this restriction to allow for some FTF communication as long as the majority of interaction occurs electronically. It remains unclear, though, what proportion of electronic communication is sufficient for a team to be classified as virtual [15]. Since our study focuses on ad-hoc teams facing a specific task, the usage of an experimental setting was appropriate. Yet, as a future direction, it is recommended to study organizational teams working on "real-world" tasks.

Future studies in this field can assist in improving virtual team compliance management and outcomes, as their findings can improve compliance among virtual team members, a concept that is used more and more routinely in today's global networking information era. 
Moreover, other behavioral characteristics of teamwork (e.g., information exchange level, leadership, time to achieve a solution) can also be studied based on similar experimental approaches. Another avenue for further research is transforming the experiment from undergraduate students to employees of organizations in order to validate whether there is similarity between the results.

\section{Conflicts of Interest}

The authors declare no conflicts of interest regarding the publication of this paper.

\section{References}

[1] Ilgen, D.R. (1999) Teams Embedded in Organizations. American Psychologist, 54, 129-139. https://doi.org/10.1037/0003-066X.54.2.129

[2] Boyett, J.H. and Conn, H.P. (1992) Workplace 2000: The Revolution Reshaping. Plume.

[3] Webster, J.D. and Staples, S. (2006) Comparing Virtual Teams to Traditional Teams: An Identification of New Research Opportunities. In: Martocchio, J.J., Ed., Research in Personnel and Human Resources Management, Vol. 25, Emerald Group Publishing Limited, Bingley, 181-215. https://doi.org/10.1016/S0742-7301(06)25005-9

[4] Caya, O., Mortensen, M. and Pinsonneault, A. (2013) Virtual Teams Demystified: An Integrative Framework for Understanding Virtual Teams. International Journal of e-Collaboration, 9, 1-33. https://doi.org/10.4018/jec.2013040101

[5] Shwartz-Asher, D. (2014) Are Virtual Team Members Compliant? 2014 International Conference on E-Commerce and Contemporary Economic Development, Hong Kong, 7-8 June 2014, 8-14.

[6] Cramton, C.D. (2001) The Mutual Knowledge Problem and Its Consequences in Geographically Dispersed Teams. Organization Science, 12, 346-371. https://doi.org/10.1287/orsc.12.3.346.10098

[7] Espinosa, J.A., Slaughter, S.A., Kraut, R.E. and Herbsleb, J.D. (2007) Team Knowledge and Coordination in Geographically Distributed Software Development. Journal of Management Information Systems, 24, 135-169. https://doi.org/10.2753/MIS0742-1222240104

[8] Jarvenpaa, S.L., Knoll, K. and Leidner, D.E. (1998) Is Anybody out There? Antecedents of Trust in Global Virtual Teams. Journal of Management Information Systems, 14, 29-64. https://doi.org/10.1080/07421222.1998.11518185

[9] Maznevski, M.L. and Chudoba, K.M. (2000) Bridging Space over Time: Global Virtual Team Dynamics and Effectiveness. Organization Science, 11, 473-492. https://doi.org/10.1287/orsc.11.5.473.15200

[10] Townsend, A.M., DeMarie, S.M. and Hendrickson, A.R. (1998) Virtual Teams: Technology and the Workplace of the Future. The Academy of Management Executive, 12, 17-29. https://doi.org/10.5465/ame.1998.1109047

[11] Berry, G.R. (2011) Enhancing Effectiveness on Virtual Teams: Understanding Why Traditional Team Skills Are Insufficient. International Journal of Business Communication, 48, 186-206. https://doi.org/10.1177/0021943610397270

[12] Maynard, M.T., Mathieu, J.E., Rapp, T.L. and Gilson, L.L. (2012) Something(s) Old and Something(s) New: Modeling Drivers of Global Virtual Team Effectiveness. 
Journal of Organizational Behavior, 33, 342-365. https://doi.org/10.1002/job.1772

[13] Hoch, J.E. and Kozlowski, S.W.J. (2014) Leading Virtual Teams: Hierarchical Leadership, Structural Supports, and Shared Team Leadership. Journal of Applied Psychology, 99, 390-403. https://doi.org/10.1037/a0030264

[14] Sivunene, A. (2006) Strengthening Identification with the Team in Virtual Teams: The Leaders' Perspective. Group Decision and Negotiation, 15, 345-366. https://doi.org/10.1007/s10726-006-9046-6

[15] Martins, L.L., Gilson, L.L. and Maynard, M.T. (2004) Virtual Teams: What Do We Know and Where Do We Go from Here? Journal of Management, 30, 805-835. https://doi.org/10.1016/j.jm.2004.05.002

[16] Furumo, K.J. and Michael, P. (2007) Gender-Based Communication Styles, Trust, and Satisfaction in Virtual Teams. Journal of Information, Information Technology, and Organizations, 2, 47-60. https://doi.org/10.28945/138

[17] Huang, W.W., Wei, K.-K., Watson, R.T. and Tan, B.C.Y. (2003) Supporting Virtual Team-Building with a GSS: An Empirical Investigation. Decision Support Systems, 34, 359-367. https://doi.org/10.1016/S0167-9236(02)00009-X

[18] Warkentin, M., Sayeed, L. and Hightower, R. (1997) Virtual Teams versus Face-to-Face Teams: An Exploratory Study of a Web-Based Conference System. Decision Sciences, 28, 975-996. https://doi.org/10.1111/j.1540-5915.1997.tb01338.x

[19] Olson, J. and Teasley, S. (1996) Groupware in the Wild: Lessons Learned from a Year of Virtual Collocation. Proceedings of the ACM Conference, Denver, 419-427. https://doi.org/10.1145/240080.240353

[20] Bouas, K.S. and Arrow, H. (1996) The Development of Group Identity in Computer and Face-to-Face Groups with Membership Change. CSCW, 4, 153-178. https://doi.org/10.1007/BF00749745

[21] Weisband, S. and Atwater, L. (1999) Evaluating Self and Others in Electronic and Face-to-Face Groups. Journal of Applied Psychology, 84, 632-639. https://doi.org/10.1037/0021-9010.84.4.632

[22] Burke, K. and Chidambaram, L. (1996) Do Mediated Contexts Differ in Information Richness? A Comparison of Collocated and Dispersed Meetings. Proceedings of the 29th Annual Hawaii International Conference on System Sciences, Hawaii, 92-101. https://doi.org/10.1109/HICSS.1996.493180

[23] Cramton, C.D. and Webber, S.S. (1999) Modeling the Impact of Geographic Dispersion on Work Teams. Working Paper, George Mason University, Washington DC.

[24] Gruenfeld, D.H., Mannix, E.A., Williams, K.Y. and Neale, M.A. (1996) Group Composition and Decision Making: How Member Familiarity and Information Distribution Affect Process and Performance. Organizational Behavior and Human Decision Processes, 67, 1-16. https://doi.org/10.1006/obhd.1996.0061

[25] Thompson, L.F. and Coovert, M.D. (2003) Teamwork Online: The Effects of Computer Conferencing on Perceived Confusion, Satisfaction, and Postdiscussion Accuracy. Group Dynamics: Theory, Research, and Practice, 7, 135-151. https://doi.org/10.1037/1089-2699.7.2.135

[26] Maruping, L.M. and Agarwal, R. (2004) Managing Team Interpersonal Processes through Technology: A Task-Technology Fit Perspective. Journal of Applied Psychology, 89, 975-990. https://doi.org/10.1037/0021-9010.89.6.975

[27] Straus, S.G. and McGrath, J.E. (1994) Does the Medium Matter? The Interaction of Task Type and Technology on Group Performance and Member Reactions. Journal 
of Applied Psychology, 79, 87-98. https://doi.org/10.1037/0021-9010.79.1.87

[28] Andres, H.P. (2002) A Comparison of Face-to-Face and Virtual Software Development Teams. Team Performance Management. An International Journal, 8, 39-48. https://doi.org/10.1108/13527590210425077

[29] Burke, K., Aytes, K. and Chidambaram, L. (2001) Media Effects on the Development of Cohesion and Process Satisfaction in Computer-Supported Workgroups. Information Technology and People, 14, 122-141. https://doi.org/10.1108/09593840110397894

[30] Baker, G. (2002) The Effects of Synchronous Collaborative Technologies on Decision Making: A Study of Virtual Teams. Information Resources Management Journal, 15, 79-93. https://doi.org/10.4018/irmj.2002100106

[31] Olson, J., Olson, G. and Meader, D. (1997) Face-to-Face Group Work Compared to Remote Group Work with and without Video. In: Finn, K., Sellen, A. and Wilbur, S., Eds., Video-Mediated Communication, Lawrence Erlbaum Associates, Mahwah, 157-172.

[32] Vaughan, G.M. and Hogg, M.A. (2011) Social Psychology. 6th Edition, Pearson Australia, Frenchs Forest.

[33] Crutchfield, R. (1955) Conformity and Character. American Psychologist, 10, 191-198. https://doi.org/10.1037/h0040237

[34] Song, G., Ma, Q., Wu, F. and Li, L. (2012) The Psychological Explanation of Conformity. Social Behavior and Personality, 40, 1365-1372.

https://doi.org/10.2224/sbp.2012.40.8.1365

[35] Cadsby, B., Maynes, E., Umashanker, V. and Trivedi, C. (2006) Tax Compliance and Obedience to Authority at Home and in the Lab: A New Experimental Approach. Experimental Economics, 9, 343.

https://doi.org/10.1007/s10683-006-7053-8

[36] Russell, N.J.C. and Gregory, R.J. (2011) Spinning an Organizational "Web of Obligation"? Moral Choice in Stanley Milgram's “Obedience” Experiments. American Review of Public Administration, 41, 495-518. https://doi.org/10.1177/0275074010384129

[37] Milgram, S. (1963) Behavioral Study of Obedience. Journal of Abnormal and Social Psychology, 67, 371-378. https://doi.org/10.1037/h0040525

[38] Bandura, A. (1999) Moral Disengagement in the Perpetration of Inhumanities. Personality and Social Psychology Review, 3, 193-209. https://doi.org/10.1207/s15327957pspr0303_3

[39] Interligi, L. (2010) Compliance Culture: A Conceptual Framework. Journal of Management and Organization, 16, 235-249. https://doi.org/10.1017/S1833367200002157

[40] Hu, Q., Dinev, T., Hart, P. and Cooke, D. (2012) Managing Employee Compliance with Information Security Policies: The Critical Role of Top Management and Organizational Culture. Decision Sciences, 43, 615. https://doi.org/10.1111/j.1540-5915.2012.00361.x

[41] Alanazi, F.M. and Rodrigues, A. (2003) Power Bases and Attribution in Three Cultures. The Journal of Social Psychology, 143, 375-395. https://doi.org/10.1080/00224540309598451

[42] Wright, T. (2013) Information Culture in a Government Organization. Records Management Journal, 23, 14-36. https://doi.org/10.1108/09565691311325004

[43] Bradner, E., Mark, G. and Hertel, T.D. (2005) Team Size and Technology Fit: Par- 
ticipation, Awareness, and Rapport in Distributed Teams. Professional Communication, 48, 68-77. https://doi.org/10.1109/TPC.2004.843299

[44] Hofner, S.D. (1996) Productive Behaviours of Global Business Teams. International Journal of Intercultural Relations, 20, 227-259. https://doi.org/10.1016/0147-1767(95)00043-7

[45] DeSanctis, G., Wright, M. and Jiang, L. (2001) Building a Global Learning Community. Communications of the ACM, 44, 80-82. https://doi.org/10.1145/501317.501352

[46] Iacono, C.S. and Weisband, S. (1997) Developing Trust in Virtual Teams. Proceedings of the 30th Hawaii International Conference on System Sciences, Maui, 7-10 January 1997, Vol. 2, 412-420. https://doi.org/10.1109/HICSS.1997.665615

[47] Hause, M., Last, M., Almstrum, V. and Woodroffe, M. (2001) Interaction Factors in Software Development Performance in Distributed Student Teams in Computer Science. Proceedings of the ACM Conference, Boulder, 69-72. https://doi.org/10.1145/377435.377477

[48] Graetz, K., Boyle, E., Kimble, C., Thompson, P. and Garloch, J. (1998) Information Sharing in Face-to-Face, Teleconferencing, and Electronic Chat Groups. Small Groups Research, 29, 714-743. https://doi.org/10.1177/1046496498296003

[49] Lebie, L., Rhoades, J.A. and McGrath, J.E. (1996) Interaction Process in Computer-Mediated and Face-to-Face Groups. CSCW, 4, 127-152.

https://doi.org/10.1007/BF00749744

[50] Siegel, J., Dubrovsky, V., Kiesler, S. and McGuire, T.W. (1986) Group Processes in Computer-Mediated Communication. Organizational Behavior and Human Decision Processes, 37, 157-188. https://doi.org/10.1016/0749-5978(86)90050-6

[51] Powell, A., Piccoli, G. and Ives, B. (2004) Virtual Teams: A Review of Current Literature and Directions for Future Research. Database for Advances in Information Systems, 35, 6-37. https://doi.org/10.1145/968464.968467

[52] Burke, K. and Chidambaram, L. (1995) Developmental Differences between Distributed and Face-to-Face Groups in Electronically Supported Meeting Environments: An Exploratory Investigation. Group Decision and Negotiation, 4, 213-223. https://doi.org/10.1007/BF01384689

[53] Strijbos, J.W., Martens, R.L., Jochems, W.M.G. and Broers, N. (2004) The Effect of Functional Roles of Group Efficiency: Using Multilevel Modeling and Content Analysis to Investigate Computer-Supported Collaboration in Small Groups. Small Group Research, 35, 195-229. https://doi.org/10.1177/1046496403260843

[54] Ahuja, M.K., Galletta, D.F. and Carley, K.M. (2003) Individual Centrality and Performance in Virtual R\&D Groups: An Empirical Study. Management Science, 49, 21-38. https://doi.org/10.1287/mnsc.49.1.21.12756

[55] Potter, R.E. and Balthazard, P.A. (2002) Understanding Human Interaction and Performance in the Virtual Team. Journal of Information Technology Theory and Application, 4, 1-23.

[56] Bunderson, J.S. and Sutcliffe, K.M. (2002) Comparing Alternative Conceptualizations of Functional Diversity in Management Teams: Process and Performance Effects. Academy of Management Journal, 45, 875-894. https://doi.org/10.2307/3069319

[57] Cummings, J.N. (2004) Work Groups, Structural Diversity, and Knowledge Sharing in a Global Organization. Management Science, 50, 352-364.

https://doi.org/10.1287/mnsc.1030.0134 
[58] Maier, N.R.F. (1967) Assets and Liabilities in Group Problem Solving. Psychology Review, 74, 239-249. https://doi.org/10.1037/h0024737

[59] Johnson, S.C., Suriya, C., Yoon, S.W., Berrett, J.V. and La Fleur, J. (2002) Team Development and Group Processes of Virtual Learning Teams. Computers and Education, 39, 379-393. https://doi.org/10.1016/S0360-1315(02)00074-X

[60] Weisband, S.P., Schneider, S.K. and Connolly, T. (1995) Computer-Mediated Communication and Social Information: Status Salience and Status Differences. The Academy of Management Journal, 38, 1124-1151. https://doi.org/10.2307/256623

[61] Kayworth, T. and Leidner, D. (2001) Leadership Effectiveness in Global Virtual Teams. Journal of Management Information Systems, 18, 7-40. https://doi.org/10.1080/07421222.2002.11045697

[62] Kim, Y., Hiltz, S.R. and Turoff, M. (2002) Coordination Structures and System Restrictiveness in Distributed Group Support Systems. Group Decision and Negotiation, 11, 379-404. https://doi.org/10.1023/A:1020492305910

[63] Tyran, K.L., Tyran, C.K. and Shepherd, M. (2003) Exploring Emerging Leadership in Virtual Teams. In: Gibson, C.B. and Cohen, S.G., Eds., Virtual Teams That Work, Jossey-Bass, San Francisco, 183-195.

[64] Yoo, Y. and Alavi, M. (2004) Emergent Leadership in Virtual Teams: What Do Emergent Leaders Do? Information and Organization, 14, 27-58. https://doi.org/10.1016/j.infoandorg.2003.11.001

[65] Fjermestad, J. and Hiltz, S.R. (1998) An Assessment of Group Support Systems Experimental Research Methodology and Results. Journal of Management Information Systems, 15, 7-149. https://doi.org/10.1080/07421222.1998.11518216

[66] Souren, P., Samarah, I.M., Seetharaman, P. and Mykytyn, P.P. (2005) An Empirical Investigation of Collaborative Conflict Management Style in Group Support System-Based Global Virtual Teams. Journal of Management Information Systems, 21, 185-222. https://doi.org/10.1080/07421222.2004.11045809

[67] Shuman, L., Besterfield-Sacre, M. and McGourty, J. (2005) The ABET "Professional Skills"-Can They Be Taught? Can They Be Assessed? Journal of Engineering Education, 94, 41-55. https://doi.org/10.1002/j.2168-9830.2005.tb00828.x

[68] Stewart, D.D. and Stasser, G. (1995) Expert Role Assignment and Information Sampling during Collective Recall and Decision Making. Journal of Personality and Social Psychology, 69, 619-628. https://doi.org/10.1037/0022-3514.69.4.619

[69] Mancia, G., Bertinieri, G., Grassi, G., Parati, G., Pomidossi, G. and Ferrari, A. (1983) Effects of Blood-Pressure Measurement by the Doctor on the Patient's Blood Pressure and Heart Rate. The Lancet, 322, 695-698. https://doi.org/10.1016/S0140-6736(83)92244-4 


\section{Appendix A}

The survey questions measured compliance:

1) My team appointed a chairperson.

Yes/No

2) If you answered "Yes" on Q1, please write the name of the chairperson:

3) My team appointed roles other than chairperson.

Yes /No

4) If you answered "Yes" on Q3, please write the roles and the name of the team members who filled the roles:

5) My team worked by process.

Yes/No

6. If you answered "Yes" on Q5, please specify the process' stages you worked according to: 